\title{
Pinning Synchronization of Linear Complex Coupling Synchronous Generators Network of Hydroelectric Generating Set
}

\author{
Xuefei Wu \\ School of Computer Engineering, Shenzhen Polytechnic, Shenzhen 518055, China \\ Correspondence should be addressed to Xuefei Wu; wuxuefei@szpt.edu.cn
}

Received 28 May 2014; Accepted 21 August 2014; Published 25 September 2014

Academic Editor: Chuandong Li

Copyright (C) 2014 Xuefei Wu. This is an open access article distributed under the Creative Commons Attribution License, which permits unrestricted use, distribution, and reproduction in any medium, provided the original work is properly cited.

A novel linear complex system for hydroturbine-generator sets in multimachine power systems is suggested in this paper and synchronization of the power-grid networks is studied. The advanced graph theory and stability theory are combined to solve the problem. Here we derive a sufficient condition under which the synchronous state of power-grid networks is stable in disturbance attenuation. Finally, numerical simulations are provided to illustrate the effectiveness of the results by the IEEE 39 bus system.

\section{Introduction}

Types of grid electricity generation have thermal power generation types, hydropower, wind power, and solar photovoltaic power generation, and there are mainly thermal power generation and hydropower in our country. Combined power generation is the problem the power plants are most concerned with in the power generation process. That is to say, power plants generating electricity need to be incorporated into the power grid to run. As most regional power supply and power generation are separately controlled by power plants and substations, power plants need to obey the scheduling, power supply bureau allowed the electricity are fed into the power grid, power plants can generate electricity. After that, excess electricity can be used by other users and if electricity generated by plant power is insufficient, the large power in the power grid can be used as a supplement. It would not be that off-grid power and self-financing. So how to make each generator successful to incorporate into the power grid is vital to the stability of the power system in entire region. In general, combined power generation must meet several requirements: voltage equal; frequency stability; and same phase. Missing any of these conditions is likely to lead to loss of synchronization of the entire generator set and then spread to the relevant local grid. Accordingly, this chapter focuses on that when synchronous generators are subject to random noise, how to quickly return to a new equilibrium and to synchronize again.

In the aspect of ensuring stability of the power system, power systems engineering experts and scholars have done a lot of research work, such as the authors of [1] discuss the self-synchronization of the power system and enhanced synchronization capability approach; a novel nonequilibrium complete synchronization condition of dynamical smart grid was proposed, whose results have improved synchronization conditions in [2]. The authors of [3] studied synchronization of the natural frequency bimodal distribution of complex power networks, and derived synchronization coupling critical condition. At the same time, the research results are applied to the European interconnected high-voltage power systems; literature [4] uses a coarse-grained scale model to study the self-organizing network synchronization power system model; they found that the more decentralized network of its dynamical behavior of nodes was, the more sensitive the random disturbance was, the more robust its topology was; literature [5] uses orthogonal decomposition method and Galerkin method to explore New Zealand's electricity network global instability, which can help to predict the real global power network instability. Ph. D. thesis $[6,7]$ studied the conditions of the electricity network synchronization. 


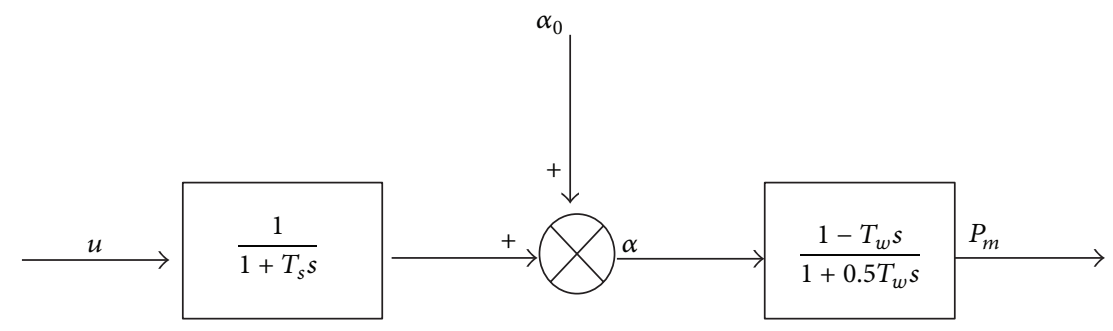

FIGURE 1: Hydroturbine speed control system block diagram.

Based on the above, we put the hydroelectric power system as the research object and discussed in detail the incorporate into the power grid of hydroelectric synchronous generators. The basic principles of hydroelectric system (hydroelectric power) are taking advantage of water flow of those rivers and lakes in high and with potential energy flow to the lower, the potential energy is converted into kinetic energy contained there in the turbine, and the turbine is regarded as the driving force to promote the generator to produce electricity. Here, we focus on the hydrogrid synchronization, namely, the voltage, frequency, and phase of all the generators connecting with each other realizing synchronization and incorporating into the power grid. In the network comprising $n$ alternators, the synchronization status can be described as follows:

$$
\dot{d}_{1}=\dot{d}_{2}=\cdots=\dot{d}_{n}
$$

where $\delta_{i}=\delta_{i}(t)$ denotes the rotation phase of the $i$ generator and $\cdot$ denotes time derivative.

We take the hydrogroup in some power plant as a small network which is composed by several synchronous generator (node) and the power transmission line (edge) connected to each other, referred to as hydrogroup synchronous generator network. Our purpose is to verify that the conditions of synchronous generators remain stable.

We will start with the following equation of motion:

$$
\frac{2 H_{i}}{\omega_{R}} \frac{d^{2} \delta_{i}}{d t^{2}}=P_{m i}-P_{e i},
$$

namely, swing equation. This swing equation describes the dynamic characteristics of the generator $i$ based on the current equation. The parameters $H_{i}$ are the inertia constant of the generator, $\omega_{R}$ is the rated angular speed of the system, $2 H_{i} / \omega_{R}$ means that all rotational inertia of rotating mass attached on the rotor shaft, the unit is $\mathrm{kg} \cdot \mathrm{m}^{2} ; \delta_{i}$ is the mechanical torque angle (subscript) with respect to synchronous rotation reference axis, the unit is $\mathrm{rad} ; P_{m i}$ is mechanical power provided by the generator, $P_{e i}$ is the generator load power in the network. In balance point, $P_{m i}=$ $P_{e i}$ and the frequency $\omega_{i} \equiv \dot{\delta}_{i}$, for all $i$, and frequencies will maintain equality with a constant; in (1), for frequency synchronization, this is a valid condition.

When the system is running at steady load conditions, due to the failure or fluctuation changes $P_{e i}$ will change. According to (2), once the system changes, the difference between the load power and mechanical power is to be compensated by the increase or decrease of the rotor angular momentum which is generated by the generator rotor. The changes in angular momentum will not remove the difference between $P_{m i}$ and $P_{e i}$ and only compensated for the difference until synchronization. Although when entering the transient period, the synchronization will be lost, but in the total process, the generator will realize synchronization by again adjusting its voltage phase angle; Each generator in power network is how to realize synchronization? Below, we will take the actual hydropower model as an example for research.

\section{Preparation and Model Description}

Hydroelectric work with the aid of hydroturbines which connect directly to the synchronous generator, when considering the impact of nonplastic water hammer, hydroturbine speed control system block diagram and description is in Figure 1 [9].

$1+T_{s} s$ is water-gate and the servo system, $T_{s}$ is time constant of a hydroturbine opening control servo motor, $\left(1-T_{w} s\right) /\left(1+0.5 T_{w} s\right)$ is hydroturbine model, $T_{w}$ is the diversion system inertia time constant, $u$ is the amount of input control, $\alpha_{0}$ is the reference value of the hydroturbine vanes, $\alpha$ is the guide vane opening, and $P_{m}$ is hydroturbine output mechanical power. We assume that an excellent excitation controller has been adopted, so as to maintain a constant potential in $q$ axis in the whole dynamic process. The hydroelectric system mainly consists of two parts, hydroturbine and its speed control system model and the generator model. According to Figure 1, we can derive single dynamic model of hydroturbine and its speed control system model:

$$
\begin{gathered}
\dot{P}_{m}=\frac{2}{T_{w}}\left[-P_{m}+\alpha-T_{w} \dot{\alpha}\right], \\
\dot{\alpha}=\frac{1}{T_{s}}\left(-\alpha+\alpha_{0}+u\right) .
\end{gathered}
$$

And single synchronous generator rotor dynamic model is

$$
\begin{gathered}
\dot{\delta}=\omega \\
\dot{\omega}=\frac{\omega_{R}}{2 H}\left[P_{m}-P_{e}-D \omega\right],
\end{gathered}
$$

where $\delta$ is the rotation phase of the generator, $\omega$ is the rotor speed, and $\omega_{R}=2 \pi f_{0}, f_{0}=60 \mathrm{~Hz} . D$ is mechanical damping 
coefficient, $H$ is the inertia time constant of the rotor. $P_{e}$ is the load power (the electric power of generator output), whereby we can get four-order hydroelectric system dynamic model $[5,9]$ :

$$
\begin{aligned}
& \frac{d \delta_{i}}{d t}=\omega_{i} \\
& \frac{d \omega_{i}}{d t}=\frac{\pi f_{0}}{H_{i}}\left\{-D_{i} \omega_{i}+P_{m i}-G_{i i} E_{i}^{2}\right. \\
& -\sum_{j=1, j \neq i}^{n}\left[E_{i} E_{j} G_{i j} \cos \left(\delta_{i}-\delta_{j}\right)\right. \\
& \left.\left.+S_{i j} \sin \left(\delta_{i}-\delta_{j}\right)\right]\right\} \\
& \frac{d P_{m i}}{d t}=\frac{2}{T_{w i}}\left[-P_{m i}+\alpha_{i}-T_{w} \dot{\alpha}_{i}\right], \\
& \frac{d \alpha_{i}}{d t}=\frac{1}{T_{s i}}\left(-\alpha_{i}+\alpha_{0 i}+u_{i}\right) .
\end{aligned}
$$

$x_{i}=\left[\delta_{i}, \omega_{i}, P_{m i}, \alpha_{i}\right] \in \mathbb{R}^{4}$ is the state variable of the $i$ generator and $f\left(x_{i}, t\right)=\left[f_{1}\left(x_{i}\right), f_{2}\left(x_{i}\right), f_{3}\left(x_{i}\right), f_{4}\left(x_{i}\right)\right]: \mathbb{R}^{4} \times$ $[0,+\infty) \rightarrow \mathbb{R}^{4}$ is a continuous mapping as follows:

$$
f\left(x_{i}, t\right)=\left\{\begin{array}{l}
\omega_{i} \\
\frac{\pi f_{0}}{H_{i}}\left[-D_{i} \omega_{i}+P_{m i}-G_{i i} E_{i}^{2}\right] \\
\frac{2}{T_{w i}}\left[-P_{m i}+\alpha_{i}-T_{w} \dot{\alpha}_{i}\right] \\
\frac{1}{T_{s i}}\left(-\alpha_{i}+\alpha_{0 i}+u_{i}\right) .
\end{array}\right.
$$

It is the dynamic equation of isolated nodes, denoting $Y_{i j}=$ $G_{i j}+i B_{i j}$ as transmission circuit admittance, $G_{i j}$ denotes the transmit circuitry conductance, $S_{i j}$ denotes the susceptance, and $E_{i}$ denotes the node voltage of the generator; based on the above, hydroelectric generating set synchronous generator network (5) can be rewritten as a more simple form as follows:

$$
\begin{aligned}
\dot{x}_{i}(t)= & f\left(x_{i}(t), t\right)+\sum_{j=1}^{n} a_{i j} \Gamma x_{j}(t) \\
& +\sum_{j=1}^{n} b_{i j} \Gamma x_{j}(t), \quad i=1, \ldots, n,
\end{aligned}
$$

where $A=\left[a_{i j}\right] \in \mathbb{R}^{n \times n}$, when $i \neq j$, then $a_{i j}=$ $\left(\pi f_{0} / H_{i}\right) E_{i} E_{j} G_{i j} \cos \delta_{i j}^{*}, \quad a_{i j}=-\sum_{j=1, j \neq i}^{n} a_{i j}, \quad i, j=$ $1,2, \ldots, n, B=\left[b_{i j}\right] \in \mathbb{R}^{n \times n}, b_{i j}=\left(\pi f_{0} / H_{i}\right) E_{i} E_{j} S_{i j} \sin \delta_{i j}^{*}, b_{i j}=$ $-\sum_{j=1, j \neq i}^{n} b_{i j}, i, j=1,2, \ldots, n$ denote the network topology, respectively. $\delta_{i j}^{*}$ denotes the phase difference while the system is stable. Next, we will discuss the synchronization of hydroelectric generating set synchronous generator network (7). To achieve synchronization, we add a node controller in the first generator. So, hydroelectric generating set synchronous generator network pinned control can be described as follows:

$$
\begin{aligned}
\dot{x}_{1}(t)= & f\left(x_{1}(t), t\right)+c \sum_{j=1}^{n} a_{1 j} \Gamma x_{j}(t) \\
& +c \sum_{j=1}^{n} b_{1 j} \Gamma x_{j}(t)-c \varepsilon \Gamma\left(x_{1}(t)-\bar{x}(t)\right), \\
\dot{x}_{i}(t)= & f\left(x_{i}(t), t\right)+c \sum_{j=1}^{n} a_{i j} \Gamma x_{j}(t) \\
& +c \sum_{j=1}^{n} b_{i j} \Gamma x_{j}(t), \quad i=2, \ldots, n,
\end{aligned}
$$

where $c$ is the control strength and $\bar{x}(t)=(1 / n) \sum_{i=1}^{n} x_{i}(t)$.

\section{Synchronization Analysis of Hydroelectric Generating Set}

In this section, we discuss pinning a hydroelectric generating set synchronous generator network with linear delay irreducible symmetric coupling matrix.

Theorem 1. If hydroelectric generating set synchronous generator network satisfied the condition as follows:

$$
I \otimes K+\bar{A}^{s} \otimes \Gamma+B^{s} \otimes \Gamma<0
$$

where $A^{S}=\left(A+A^{T}\right) / 2$, then the controlled hydroelectric generating set synchronous generator network (8) can be globally synchronized to $\bar{x}$, where

$$
\begin{gathered}
K=\left[\begin{array}{cccc}
0 & 1 & 0 & 0 \\
0 & -\frac{\pi f_{0} D_{i}}{H} & \frac{\pi f_{0}}{H} & 0 \\
0 & 0 & -\frac{2}{T_{w}} & \frac{2}{T_{w}}+\frac{2}{T_{s}} \\
0 & 0 & 0 & -\frac{1}{T_{s}}
\end{array}\right], \\
\bar{A}=\left(\bar{a}_{i j}\right)_{n \times n}=A-\operatorname{diag}\left\{\left(1-\frac{1}{n}\right) \varepsilon, 0, \ldots, 0\right\} .
\end{gathered}
$$

Proof. Define $\Delta x_{i}(t)=x_{i}(t)-\bar{x}(t)$. Because $\sum_{j=1}^{n} a_{i j} \bar{x}(t)=0$, we can easily get

$$
\sum_{j=1}^{m} a_{i j} x_{j}(t)=\sum_{j=1}^{m} a_{i j} \Delta x_{j}(t),
$$


so the error system of hydroelectric generating set synchronous generator network (8) of can be rewritten as

$$
\begin{aligned}
\frac{d \Delta x_{1}(t)}{d t}= & f\left(x_{1}(t), t\right)-f(\bar{x}(t), t)+\sum_{j=1}^{n} a_{1 j} \Gamma \Delta x_{j}(t) \\
& +\sum_{j=1}^{n} b_{1 j} \Gamma \Delta x_{j}(t)-\left(1-\frac{1}{n}\right) \varepsilon \Gamma \Delta x_{1}(t)+J, \\
\frac{d \Delta x_{i}(t)}{d t}= & f\left(x_{i}(t), t\right)-f(\bar{x}(t), t)+\sum_{j=1}^{n} a_{i j} \Gamma \Delta x_{j}(t) \\
& +\sum_{j=1}^{n} b_{i j} \Gamma \Delta x_{j}(t)+J, \quad i=2, \ldots, n,
\end{aligned}
$$

where, $J=f(\bar{x}(t), t)-(1 / n) \sum_{i=1}^{n} f\left(x_{i}(t), t\right)$.

Take Lyapunov function

$$
V(t)=\frac{1}{2} \sum_{i=1}^{n} \Delta x_{i}^{T}(t) \Delta x_{i}(t)
$$

and define $\Delta x(t)=\left(\Delta x_{1}^{T}(t), \ldots, \Delta x_{n}^{T}(t)\right)^{T}, i=1, \ldots, n$. Now we calculate

$$
\begin{aligned}
& \left.\dot{V}(t)\right|_{(12)} \\
& =\sum_{i=1}^{n} \Delta x_{i}^{T}(t) \\
& \times\left[f\left(x_{i}(t), t\right)-f(\bar{x}(t), t)\right. \\
& \left.+\sum_{j=1}^{n} \bar{a}_{i j} \Gamma \Delta x_{j}(t)+\sum_{j=1}^{n} b_{i j} \Gamma \Delta x_{j}(t)+J\right] \\
& =\sum_{i=1}^{n} \Delta x_{i}^{T}(t) K \Delta x_{i}(t) \\
& +\sum_{i=1}^{n} \Delta x_{i}^{T}(t) \sum_{j=1}^{n} \bar{a}_{i j} \Gamma \Delta x_{j}(t) \\
& +\sum_{i=1}^{n} \Delta x_{i}^{T}(t) \sum_{j=1}^{n} b_{i j} \Gamma \Delta x_{j}(t) \\
& =\Delta x^{T}(t)(I \otimes K) \Delta x(t)+\Delta x^{T}(t)(\bar{A} \otimes \Gamma) \Delta x(t) \\
& +\Delta x^{T}(t)(B \otimes \Gamma) \Delta x(t) \\
& =\Delta x^{T}(t)(I \otimes K+\bar{A} \otimes \Gamma+B \otimes \Gamma) \Delta x(t) .
\end{aligned}
$$

We know, based on Theorem 1, that $\bar{A}$ is negative definite matrix; obviously, the condition (9) can be satisfied easily; therefore, we can get $\dot{V}(t)<0$. Theorem 1 is proved.

Remark 2. If the hydroelectric generating set synchronous generator network meet the condition $I \otimes K+\bar{A} \otimes \Gamma+B^{S} \otimes \Gamma<0$,

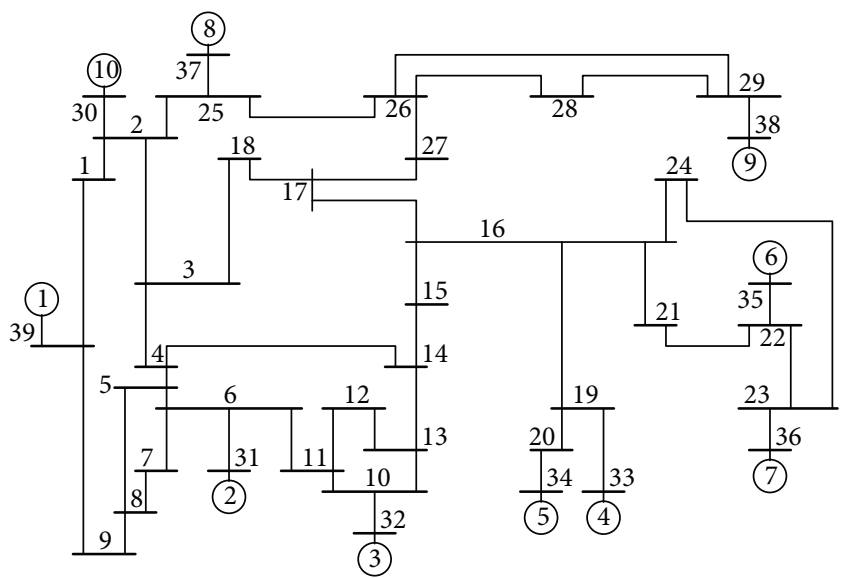

FIGURE 2: Grid network structure (which is generator nodes connected with circle, a total of 10 , and the remaining nodes are nongenerator nodes. $[2,8])$.

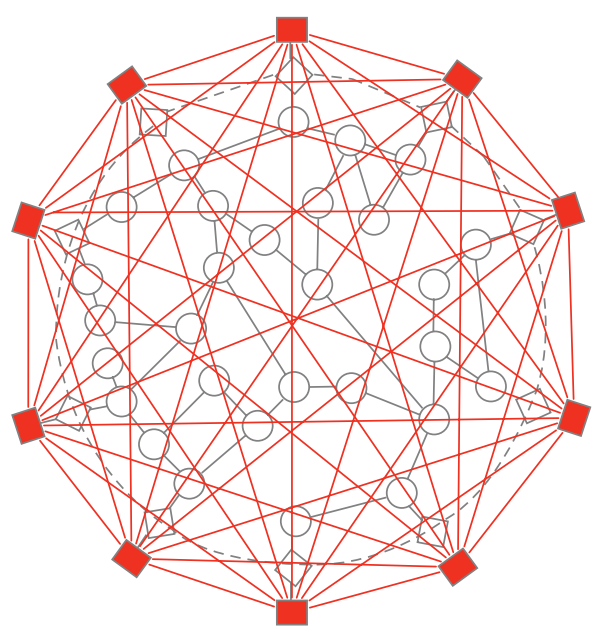

FIGURE 3: Network topology after simplifying Figure 2 by using Kron method (the 10 red nodes in the figure are the generator nodes, all links between the generator nodes. [2]).

then the controlled hydroelectric generating set synchronous generator network (8) can be globally synchronized. Here, the synchronization of network is decided by electric system itself and the network structure.

\section{Numerical Simulation}

Numerical simulation of this section, we mainly consider IEEE 39 test grid (data using $100 \mathrm{MVA}, 60 \mathrm{~Hz}$ ) [8], as shown in Figure 2 and Table 1. Figure 3 shows network topology after simplifying Figure 2 by using Kron method.

In the numerical experiments, let the nominal angular velocity $\omega_{0}=60 \mathrm{~Hz}$, the reference value of hydroturbine guide vanet $\alpha_{0}=1$, the mechanical damping coefficient $D=$ 0.5 , rotor inertia time constant $H=4$, inertia time constant of diversion system $T_{w}=4$, and hydroturbine opening control servo motor time constant $T_{s}=4$. In this paper, we let $u$ be 0 , we can verify it satisfies theorem. Each of the generator 
TABLE 1: P.S. IEEE39 power network node admittance.

\begin{tabular}{|c|c|c|}
\hline$i$ & $j$ & $y_{i j}$ \\
\hline 1 & 2 & $0.0035+0.0411 i$ \\
\hline 1 & 39 & $0.0020+0.050 i$ \\
\hline 2 & 3 & $0.0013+0.0151 i$ \\
\hline 2 & 25 & $0.0070+0.0086 i$ \\
\hline 3 & 4 & $0.0013+0.0213 i$ \\
\hline 3 & 18 & $0.0011+0.0133 i$ \\
\hline 4 & 5 & $0.00080+0.0128 i$ \\
\hline 4 & 14 & $0.00080+0.0129 i$ \\
\hline 5 & 6 & $0.00020+0.0026 i$ \\
\hline 5 & 8 & $0.00080+0.0112 i$ \\
\hline 6 & 7 & $0.00060+0.0092 i$ \\
\hline 6 & 11 & $0.00070+0.0082 i$ \\
\hline 7 & 8 & $0.00040+0.0046 i$ \\
\hline 8 & 9 & $0.0023+0.0363 i$ \\
\hline 9 & 39 & $0.0010+0.025 i$ \\
\hline 10 & 11 & $0.00040+0.0043 i$ \\
\hline 10 & 13 & $0.00040+0.0043 i$ \\
\hline 13 & 14 & $0.00090+0.0101 i$ \\
\hline 14 & 15 & $0.0018+0.0217 i$ \\
\hline 15 & 16 & $0.00090+0.0094 i$ \\
\hline 16 & 17 & $0.00070+0.0089 i$ \\
\hline 16 & 19 & $0.0016+0.0195 i$ \\
\hline 16 & 21 & $0.00080+0.0135 i$ \\
\hline 16 & 24 & $0.00030+0.0059 i$ \\
\hline 17 & 18 & $0.00070+0.0082 i$ \\
\hline 17 & 27 & $0.0013+0.0173 i$ \\
\hline 21 & 22 & $0.00080+0.014 i$ \\
\hline 22 & 23 & $0.00060+0.0096 i$ \\
\hline 23 & 24 & $0.0022+0.035 i$ \\
\hline 25 & 26 & $0.0032+0.0323 i$ \\
\hline 26 & 27 & $0.0014+0.0147 i$ \\
\hline 26 & 28 & $0.0043+0.0474 i$ \\
\hline 26 & 29 & $0.0057+0.0625 i$ \\
\hline 28 & 29 & $0.0014+0.0151 i$ \\
\hline 2 & 30 & $0.0181 i$ \\
\hline 6 & 31 & $0.050 i$ \\
\hline 6 & 31 & $0.0+0.050 i$ \\
\hline 10 & 32 & $0.0+0.020 i$ \\
\hline 12 & 11 & $0.0016+0.0435 i$ \\
\hline 12 & 13 & $0.0016+0.0435 i$ \\
\hline 19 & 20 & $0.00070+0.0138 i$ \\
\hline 19 & 33 & $0.00070+0.0142 i$ \\
\hline 20 & 34 & $0.00090+0.018 i$ \\
\hline 22 & 35 & $0.0+0.0143 i$ \\
\hline 23 & 36 & $0.00050+0.0272 i$ \\
\hline 25 & 37 & $0.00060+0.0232 i$ \\
\hline 29 & 38 & $0.00080+0.0156 i$ \\
\hline
\end{tabular}

initial speed is randomly generated by the computer (attached generator admittance between nodes); using the function

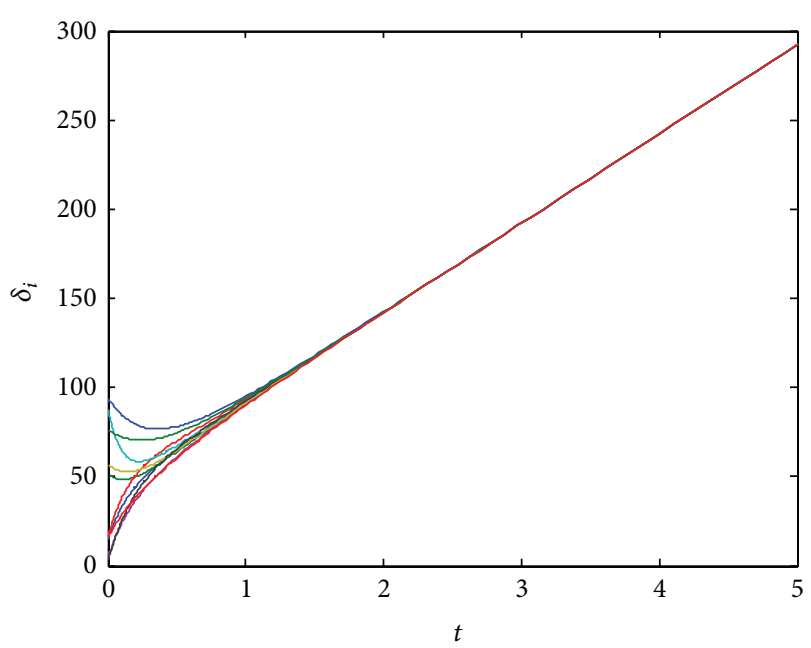

FIGURE 4: The evolution process of pinning the rotor phase of 10 generator nodes.

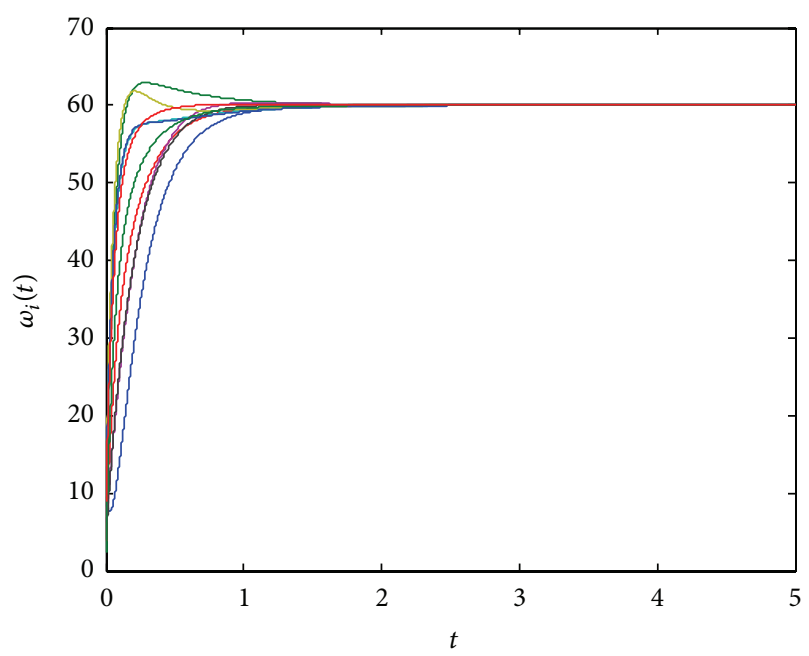

FIGURE 5: The evolution process of pinning the rotor frequency of 10 generator nodes.

ode45 in MATLAB solve (7), we can obtain the numerical results of the individual states for each node.

Figures 4 and 5 are evolution schematic diagrams with hydroelectric generating set synchronous generator network of 10 generator nodes, generator rotor phase, and the rotor frequency. Figures 6 and 7 are mechanical power for the generator and the guide vane opening states. From the figures, we can obviously find that all the generators are synchronized which indicate that the algorithm described in this chapter is valid to achieve synchronization of hydroelectric generating set synchronous generator network. 


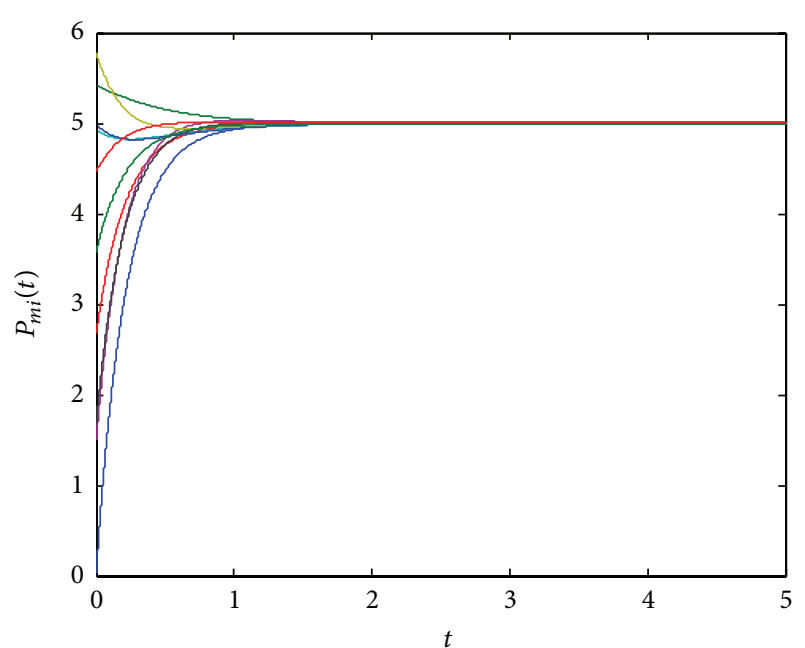

FIGURE 6: The trajectory with mechanical power of 10 generator nodes.

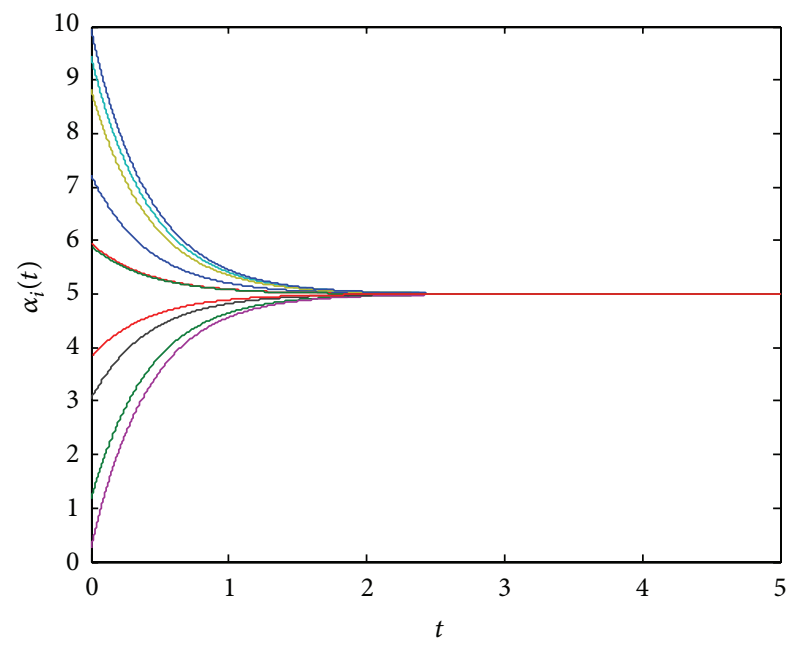

FIGURE 7: The trajectory with guide vane opening of 10 generator nodes.

\section{Conclusion}

Synchronization is a very important research field in the application of science and engineering; particularly in complex power network generator synchronizing has more application prospects. How to simplify the system pinning process is of vital significance to improve the efficiency of the system. So, based on existing work, the synchronization of hydroelectric generating set synchronous generator network is discussed. The parameters will be dimension, and the corresponding complex network model is established; we also design a linear feedback controller adding the first generator to realize the phase and frequency synchronization of hydroelectric generating set synchronous generator by using the stability theory for hydrogenerator units to realize synchronous conditions in theory. In the end, we provided some numerical simulation to verify the validity of the theorem.

\section{Conflict of Interests}

The author declares that there is no conflict of interests regarding the publication of this paper.

\section{Acknowledgments}

The author thanks the referees and the editor for their valuable comments on this paper. This work was supported by Shenzhen Basic Research Project (JCYJ20130331152625792).

\section{References}

[1] A. E. Motter, S. A. Myers, M. Anghel, and T. Nishikawa, "Spontaneous synchrony in power-grid networks," Nature Physics, vol. 9, no. 3, pp. 191-197, 2013.

[2] F. A. Dörfler, Dynamics and Control in Power Grids and Complex Oscillator Networks, University of California, 2013.

[3] S. Lozano, L. Buzna, and A. Díaz-Guilera, "Role of network topology in the synchronization of power systems," The European Physical Journal B, vol. 85, no. 7, article 231, 2012.

[4] M. Rohden, A. Sorge, M. Timme, and D. Witthaut, "Selforganized synchronization in decentralized power grids," Physical Review Letters, vol. 109, no. 6, Article ID 064101, 2012.

[5] Y. Susuki, I. Mezic, and T. Hikihara, "Global swing instability in the New England power grid model," in Proceedings of the American Control Conference (ACC '09), pp. 3446-3451, June 2009.

[6] F. Dörfler, M. Chertkov, and F. Bullo, "Synchronization in complex oscillator networks and smart grids," Proceedings of the National Academy of Sciences of the United States of America, vol. 110, no. 6, pp. 2005-2010, 2013.

[7] F. Dörfler and F. Bullo, "Synchronization in complex networks of phase oscillators: a survey," Automatica, vol. 50, no. 6, pp. 1539-1564, 2014.

[8] M. A. Pai, Energy Function Analysis for Power System Stability, Springer, New York, NY, USA, 1989.

[9] Q. Lu, Y. Sun, Y. Sun et al., "Nonlinear decentralized robust governor control for hydroturbine-generator sets in multimachine power systems," International Journal of Electrical Power and Energy System, vol. 26, no. 5, pp. 333-339, 2004. 


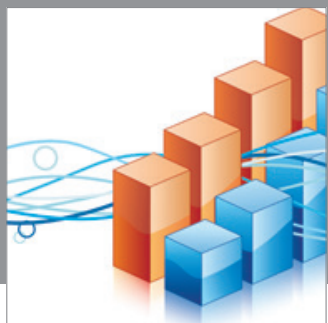

Advances in

Operations Research

mansans

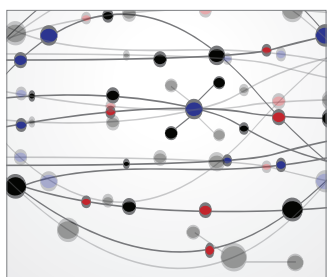

The Scientific World Journal
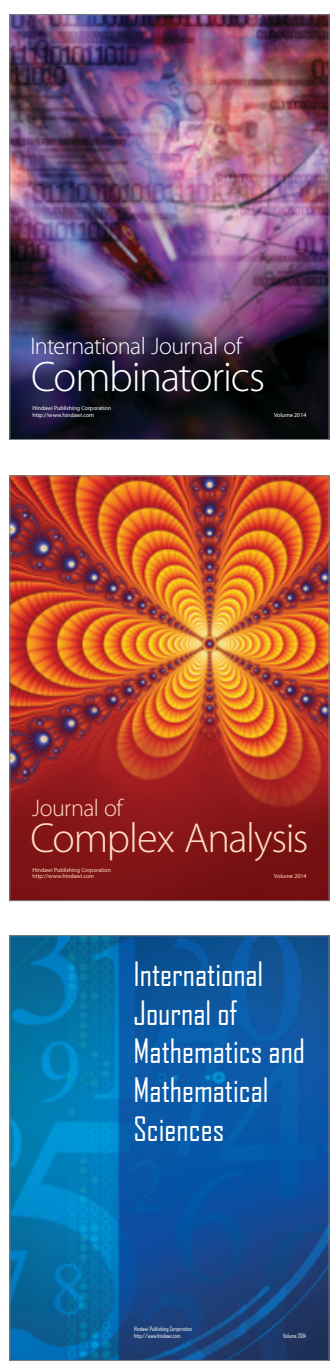
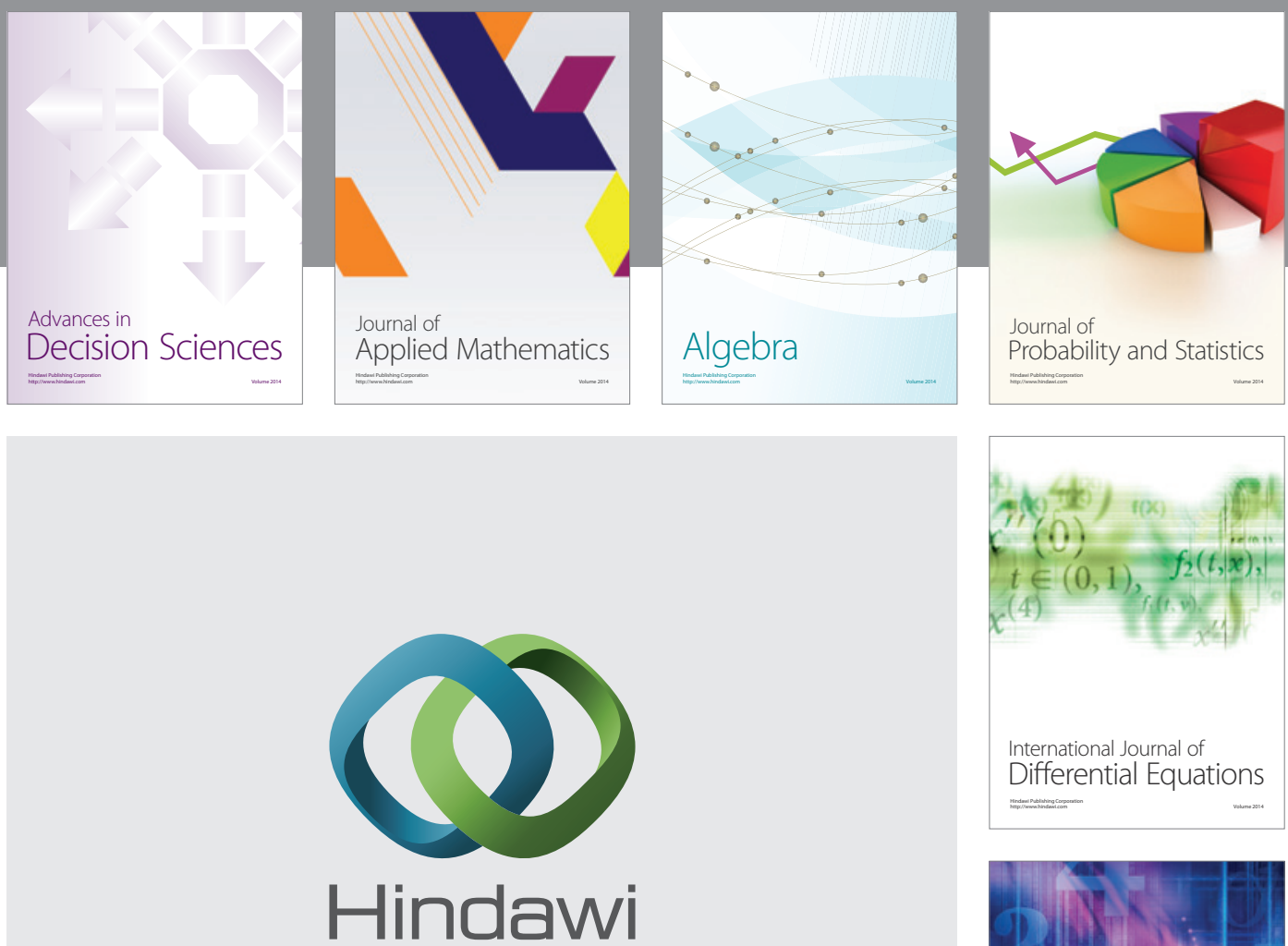

Submit your manuscripts at http://www.hindawi.com
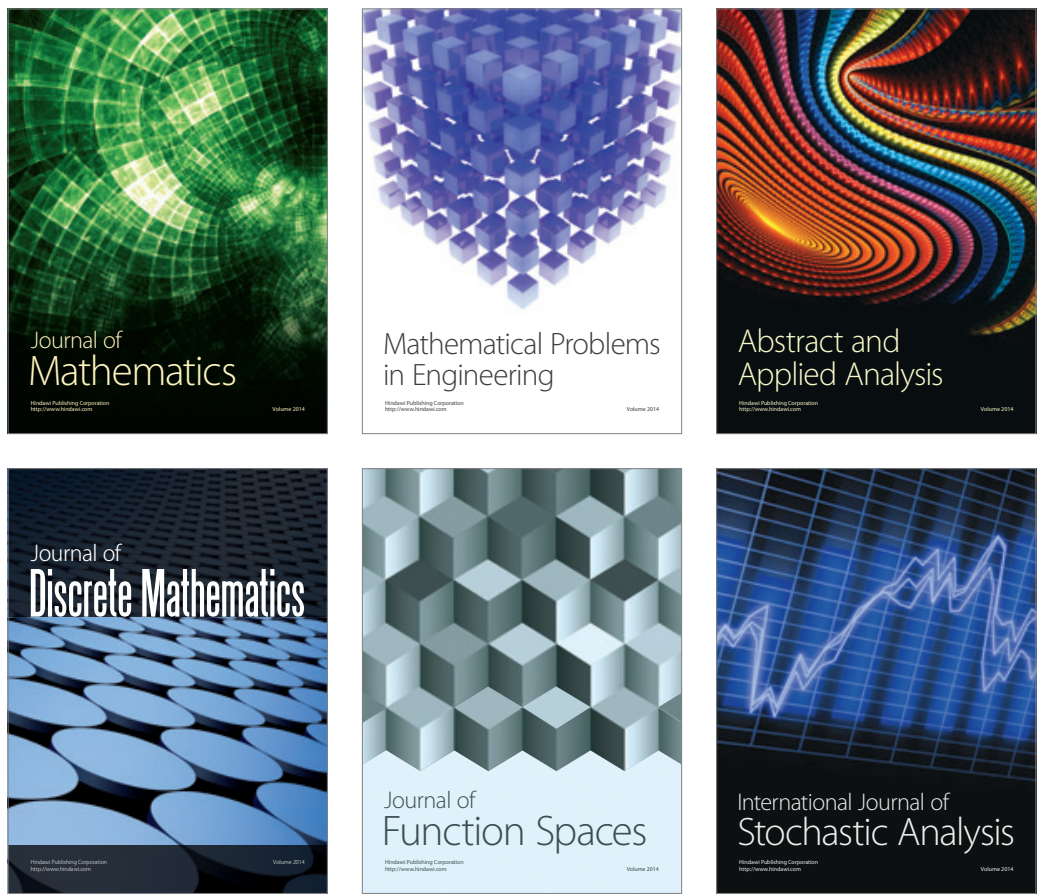

Journal of

Function Spaces

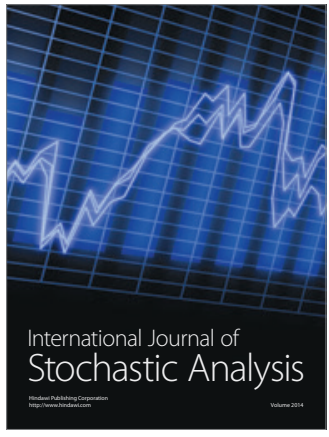

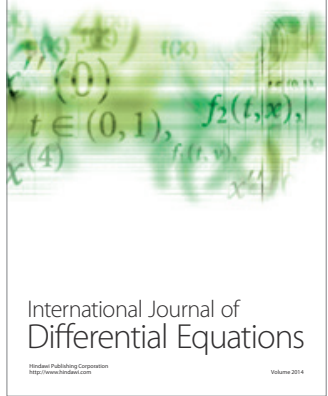
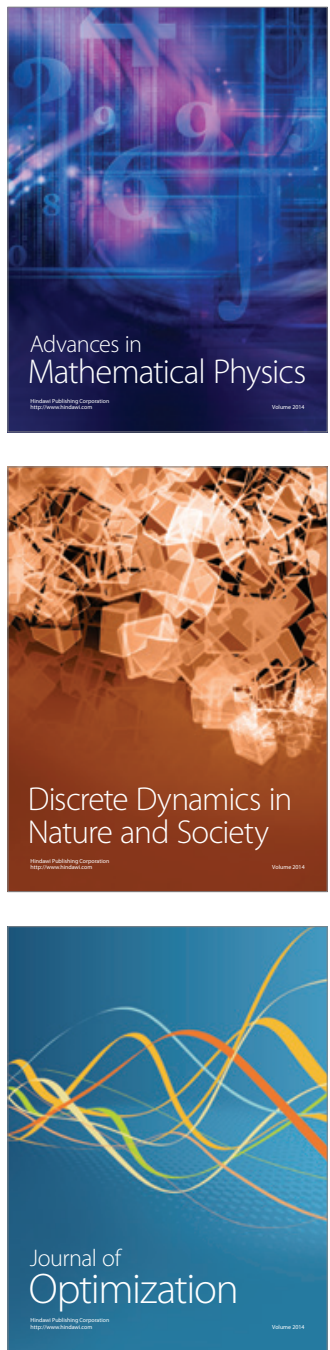